\title{
Diagnostic accuracy of circulating miR-126 for malignant pleural mesothelioma: a systematic review and meta-analysis
}

\author{
Lei Zhang ${ }^{1}$, Qianghua Zhou ${ }^{2}$, Yan-Qiu Han ${ }^{1}$, Peng Li ${ }^{1}$, Pei-Heng Ouyang ${ }^{1}$, Mei-Ying Wang ${ }^{1}$, \\ Zhi-De $\mathrm{Hu}^{1 \wedge}$
}

${ }^{1}$ Department of Laboratory Medicine, the Affiliated Hospital of Inner Mongolia Medical University, Hohhot, China; ${ }^{2}$ Laboratory Medicine Program, University Health Network, Toronto, Ontario, Canada

Contributions: (I) Conception and design: MY Wang, ZD Hu; (II) Administrative support: Q Zhou, YQ Han, P Li, PH Ouyang; (III) Provision of study materials or patients: None; (IV) Collection and assembly of data: L Zhang, ZD Hu; (V) Data analysis and interpretation: L Zhang, MY Wang, ZD Hu; (VI) Manuscript writing: All authors; (VII) Final approval of manuscript: All authors.

Correspondence to: Mei-Ying Wang. Department of Laboratory Medicine, the Affiliated Hospital of Inner Mongolia Medical University, Hohhot 010050, China. Email: 18047191049@163.com; Zhi-De Hu, MD. Department of Laboratory Medicine, the Affiliated Hospital of Inner Mongolia Medical University, Hohhot 010050, China. Email: hzdlj81@163.com.

\begin{abstract}
Background: Circulating microRNAs are novel diagnostic markers for various types of cancer. Several studies have investigated the diagnostic accuracy of circulating miR-126 for malignant pleural mesothelioma (MPM), but the results varied. Therefore, we performed a systematic review and meta-analysis to investigate the diagnostic value of circulating miR-126 for MPM.

Methods: The PubMed database was searched to identify potentially eligible studies published before October 2020. The studies investigating the diagnostic value of circulating miR-126 for MPM were included in a systematic review and meta-analysis. A bivariate model was used to pool eligible studies' sensitivity and specificity. The revised tool for the quality assessment of diagnostic accuracy studies (QUADAS-2) was used to assess eligible studies' quality.

Results: Four studies with 156 MPM patients and 459 controls were included in this systematic review and meta-analysis. The pooled diagnostic sensitivity and specificity of circulating miR-126 for MPM were 0.71 and 0.69 , respectively. A high risk of bias was observed in the domains of patient selection, index test, and flow and timing.

Conclusions: Circulating miR-126 has limited value for diagnosing MPM. Considering that the available studies have a high risk of bias, further rigorous studies are needed to assess the diagnostic value of circulating miR-126 for MPM.
\end{abstract}

Keywords: Malignant pleural mesothelioma (MPM); diagnosis; miR-126; meta-analysis

Submitted Jan 18, 2021. Accepted for publication Mar 24, 2021.

doi: $10.21037 /$ tcr-21-104

View this article at: http://dx.doi.org/10.21037/tcr-21-104

\section{Introduction}

Malignant pleural mesothelioma (MPM) is a type of cancer originating from the pleura with high mortality. MPM patients are usually diagnosed at a late stage due to lack of specific symptoms and signs at an early stage. The outcome of MPM can be improved by early detection and treatment. Currently, image-guided biopsy is the gold standard for MPM diagnosis; however, it has limitations (1), including invasiveness, observer-dependent and special training. Therefore, it is of great value to

^ ORCID: Qianghua Zhou, 0000-0001-7483-0478; Zhi-De Hu, 0000-0003-3679-4992. 
develop non-invasive and easy-to-perform diagnostic tools. Laboratory testing represents a potential diagnostic tool for MPM because it has advantages of non-invasiveness, easyto-perform and low cost. Several diagnostic biomarkers, either in pleural effusion or blood, have been identified $(2,3)$, including soluble mesothelin-related peptide (SMRP) (4), osteopontin (5), cytokeratin 19 fragment (CYFRA 21-1) $(3,6)$ and fibulin-3 (7). However, these biomarkers' diagnostic accuracy was unsatisfactory. Therefore, it is of great value to develop novel diagnostic markers for MPM (8).

MicroRNAs are a type of non-coding RNAs with a length of 18 to $25 \mathrm{bp}$. It regulates the target gene expression at the post-transcriptional level by binding to the 3' untranslated region (UTR) of mRNA. It has multiple biological functions including but not limited to cancer development, immune response, and embryogenesis (9). Previous studies indicated that circulating microRNA is stable and can be determined $(10,11)$. These circulating microRNAs can be used as diagnostic markers for various diseases. Some circulating microRNAs have been identified for diagnosing MPM, such as miR-29 (12), miR-92 (13), miR-625-3p (13) and miR-126. Among the reported circulating microRNAs, miR-126 is the most widely studied one (14). However, the diagnostic accuracy of circulating miR-126 in the available studies varied. Hence, we performed a systematic review and meta-analysis to estimate the diagnostic accuracy of circulating miR-126 for MPM. We presented the article in accordance with the Preferred Reporting Items for a Systematic Review and Meta-analysis of Diagnostic Test Accuracy Studies (PRISMA-DTA) (15) reporting checklist (available at http://dx.doi.org/10.21037/ tcr-21-104).

\section{Methods}

\section{Database and literature search}

This study was not registered previously, and its protocol was not published. We searched the PubMed database to identify potential studies published before October 1 , 2020. The literature search algorithm was: (miR-126 or microRNA-126 or miR-126-3p or microRNA-126-3p or "MIRN126 microRNA, human"[nm]) and mesothelioma. All searched studies were imported into the Endnote software for study screening.

\section{Inclusion and exclusion criteria}

The meta-analysis's inclusion criteria were: (I) studies investigating the diagnostic accuracy of circulating miR-126 for MPM; (II) sensitivity and specificity were reported, and a two-by-two table can be constructed for meta-analysis. The exclusion criteria were: (I) animal studies; (II) review, comment, editorial or letter to the editor; (III) conference abstract.

Two reviewers independently reviewed the searched studies. In the first round, we screened the studies' titles and abstracts to exclude apparently irrelevant records. A full-text screening was conducted in the second round to ascertain the remaining studies' eligibility. Any disagreement in study selection was resolved by consensus.

\section{Data extraction}

Two reviewers independently extracted the following data from the eligible studies: the first author, publication year, country, sample sizes of MPM and control, the characteristics of non-MPM, type of data collection (prospective or retrospective), consecutively enrollment, internal control for miR-126 determination, reference for diagnosing MPM, the area under the curve (AUC) of miR126 , sensitivity, specificity and threshold adopted. With the sensitivity, specificity and sample sizes of MPM and non-MPM, a two-by-two table was constructed for metaanalysis.

\section{Quality assessment}

We used the revised tool for the quality assessment of diagnostic accuracy studies (QUADAS-2) to assess eligible studies' quality (16). The QUADAS-2 tool assesses the risk of bias and applicability concerns of the eligible studies with four domains: patient selection, index test, reference, flow and timing. Any disagreement in quality assessment was resolved by consensus.

\section{Statistical analysis}

This meta-analysis was performed with the metaDTA, an interactive web-based tool for meta-analysis of diagnostic test accuracy studies (17). The statistical method used in metaDTA is the bivariate model (18). A summary receiver operating characteristic (sROC) was used to estimate the globe accuracy of circulating miR-126 (19). Because only four studies were included in this meta-analysis, subgroup analysis, sensitivity analysis, publication bias analysis, and meta-regression were not performed. 


\section{Results}

\section{Summary of the eligible studies}

Figure 1 is a flowchart of the study selection process. After an abstract and full-text screening, four studies were included in this systematic review and meta-analysis (20-23). The characteristics of these included studies are summarized in Table 1. Three studies were from Italy (20-22), and one was from Germany (23). The sample size ranged from 66 to 240 , and the total sample size of this meta-analysis was 615 , with 156 of them being MPM. The non-MPM patients in these studies included asbestosexposed subjects (AES), healthy control and non-small cell lung cancer (NSCLC). All of the studies did not enroll subjects consecutively, and they also did not report whether the circulating miR-126 level was blinded to clinicians who diagnosed MPM. The type of data collection (prospective, retrospective) was also not reported in all studies. Two

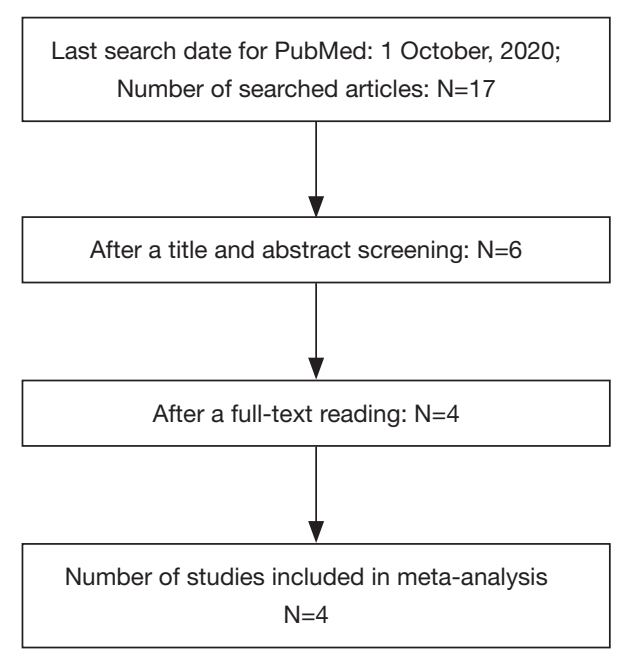

Figure 1 Flowchart of study selection. studies used U6 as the internal control in detecting circulating miR-126 with PCR $(20,23)$, while the remaining two studies used cel-miR-39 $(21,22)$. The biopsy was used as a gold standard in three studies (20-22), while one study did not report the gold standard for MPM diagnosis.

\section{Quality assessment}

Table 2 lists the quality assessment results of the included studies. Generally, the quality of the included studies was low. All studies have a high risk of patient selection because all subjects were not consecutively enrolled. There were no uniform inclusion and exclusion criteria for patient enrollment. The index test domain of all included studies has a high risk of bias because these studies used a datadriven method to define the diagnostic threshold of circulating miR-126. The flow and timing domain in three studies was labeled "high" because not all subjects received the same reference, termed as partial verification bias (24).

\section{Meta-analysis}

Table 3 shows the diagnostic accuracy of miR-126 in all included studies. Two studies reported the AUC of miR-126 was less than 0.80 , indicating that its diagnostic accuracy was moderate.

Figure 2 is a forest plot of miR-126. The sensitivity ranged from 0.59 to 0.76 , and the specificity ranged from 0.54 to 0.86 . The pooled sensitivity, specificity, negative likelihood ratio (NLR), positive likelihood ratio (PLR) and diagnostic odds ratio (DOR) were 0.71 (95\% CI: $0.63-0.77$ ), 0.69 (95\% CI: 0.56-0.80), 2.28 (95\% CI: 1.51-3.46), 0.43 (95\% CI: $0.32-0.58$ ) and 5.35 (95\% CI: 2.72-10.52), respectively. Great heterogeneity was observed across all eligible studies, with an $\mathrm{I}^{2}$ of $76 \%$ (95\% CI: $\left.48 \%-100 \%\right)$. All heterogeneity $(100 \%)$ was likely due to the threshold effect. Figure 3 is

Table 1 Summary of the eligible studies

\begin{tabular}{|c|c|c|c|c|c|c|c|c|c|}
\hline Author & Year & Country & $\begin{array}{l}\text { N (MPM/ } \\
\text { non-MPM) }\end{array}$ & $\begin{array}{l}\text { Characteristics } \\
\text { of non-MPM }\end{array}$ & $\begin{array}{l}\text { Data } \\
\text { collection }\end{array}$ & $\begin{array}{l}\text { Consecutive } \\
\text { enrollment }\end{array}$ & $\begin{array}{l}\text { Internal } \\
\text { control }\end{array}$ & $\begin{array}{l}\text { Blinded } \\
\text { interpretation }\end{array}$ & Reference \\
\hline Santarelli (20) & 2011 & Italy & $44 / 196$ & AES & NR & No & U6 & NR & Biopsy \\
\hline Santarelli (22) & 2015 & Italy & $45 / 143$ & AES, HC & NR & No & Cel miR-39 & NR & Biopsy \\
\hline
\end{tabular}

MPM, malignant pleural mesothelioma; AES, asbestos-exposed subjects; HC, healthy control; NSCLC, non-small cell lung cancer; NR, not reported. 
Table 2 Quality assessment of the eligible studies

\begin{tabular}{|c|c|c|c|c|c|c|c|}
\hline Author & \multicolumn{4}{|c|}{ Risk of bias } & \multicolumn{3}{|c|}{ Applicability concerns } \\
\hline Santarelli (20) & High & High & Low & High & Low & Low & Low \\
\hline Tomasetti (21) & High & High & Low & High & High & Low & Low \\
\hline Santarelli (22) & High & High & Low & High & High & Low & Low \\
\hline
\end{tabular}

Table 3 Diagnostic accuracy of circulating miR-126 in included studies

\begin{tabular}{lcccccccc}
\hline Author & AUC & Cut-off & Sensitivity & Specificity & TP & FN & FP & TN \\
\hline Santarelli (20) & NR & -4.5 & 0.73 & 0.74 & 32 & 12 & 51 & 145 \\
Tomasetti (21) & 0.89 & -24 & 0.70 & 0.60 & 31 & 14 & 30 \\
Santarelli (22) & 0.71 & 0.01 & 0.75 & 0.54 & 34 & 11 & 66 \\
Weber (23) & 0.76 & 30.28 & 0.59 & 0.86 & 13 & 9 & 6 \\
\hline
\end{tabular}

AUC, the area under receiver operator characteristics curve; TP, true positive; FN, false negative; FP, false positive; TN, true negative; NR, not reported.

Forest plot of sensitivity

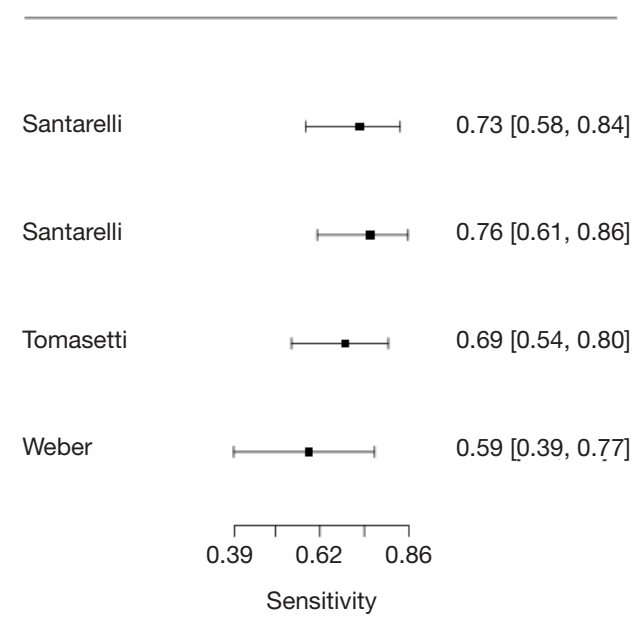

Forest plot of specificity

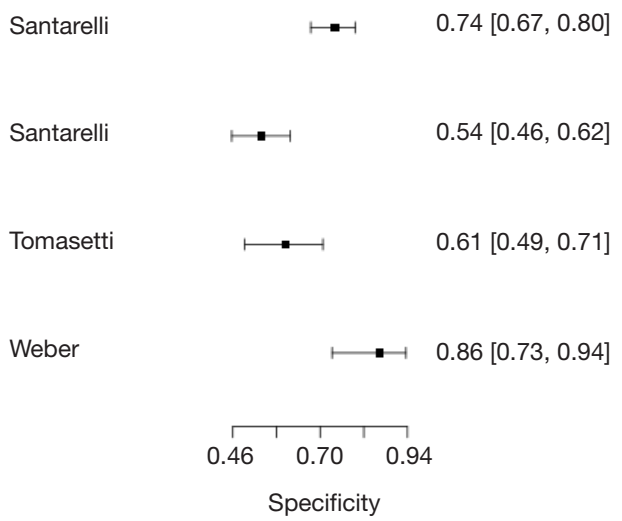

Figure 2 Forest plot of miR-126.

an sROC plot for miR-126, the AUC was 0.74 (95\% CI: $0.70-0.77)$.

Table 4 summarizes the evidence regarding the diagnostic accuracy of circulating or pleural effusion SMRP, osteopontin, fibulin-3 and miR-126 for MPM. Notably, the specificity and AUC of miR-126 were the lowest among these diagnostic markers.

\section{Discussion}

As a diagnostic tool, circulating cell-free microRNAs have gained much attention these years. In this study, we performed a systematic review and meta-analysis to evaluate the diagnostic accuracy of circulating miR-126 for MPM. We found that the diagnostic accuracy of circulating miR- 
126 was low, with both sensitivity and specificity around 0.70 and an AUC of 0.73 .

Among the available circulating diagnostic markers for MPM, SMRP (4), osteopontin (5), and fibulin-3 (7) have been widely investigated. We found that the sensitivity of circulating miR-126 was 0.71 , which is slightly higher than that of circulating SMRP and osteopontin but lower than that of fibulin-3 and PE SMRP. The specificity of miR-126 is lower than that of circulating or PE SMRP, osteopontin and fibulin-3, indicating that the diagnostic accuracy of circulating miR-126 was not superior to that of traditional biomarkers. Both sensitivity and specificity are largely affected by the threshold adopted, thus are not

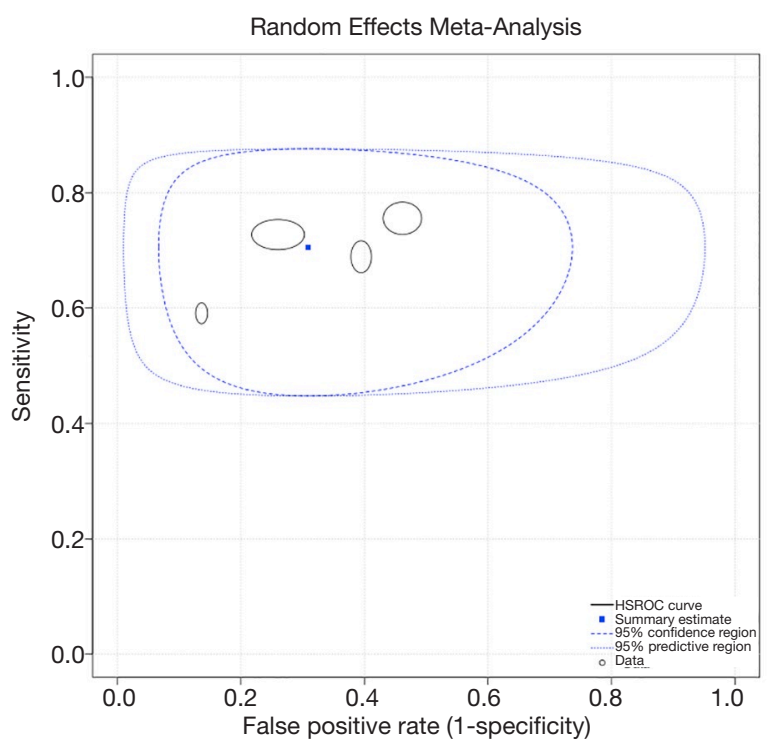

Figure 3 Summary receiver operating characteristic (sROC) of miR-126 for diagnosing malignant pleural mesothelioma. reliable metrics to estimate the diagnostic accuracy of a given index test. By contrast, AUC of sROC curve is not affected by the threshold, and thus used as a globe indicator used to estimate the diagnostic accuracy of an index test. The AUC of circulating miR-126 was 0.74 , lower than that of SMRP, osteopontin, and fibulin-3, which also support that the diagnostic accuracy of circulating miR-126 was inferior to that of these traditional biomarkers. Therefore, circulating miR-126 does not have the potential to replace these biomarkers. Future studies are needed to ascertain whether combining circulating miR-126 with these markers can improve the diagnostic accuracy of MPM.

Notably, not all of the included studies were reported following the Standards for Reporting of Diagnostic Accuracy (STARD) guideline (25). Therefore, some design details were unclear, and the corresponding domain in the QUADAS-2 tool was labeled as unknown. That means the risk of bias and applicability concerns cannot be assessed accurately. From the reported detail in the included articles, the available studies have some design weaknesses which may negatively affect the eligible studies' reliability. Subjects in some eligible studies were not consecutively enrolled with uniform inclusion and exclusion criteria. Therefore, the representativeness of the studied cohort may be affected. One study even included healthy individuals as a control, leading to an overestimation of circulating miR126 's diagnostic accuracy $(24,26)$. In addition, the threshold used to define positive and negative miR-126 was not prespecified in all studies, and none of the included studies used training and validating cohorts to estimate and validate the diagnostic accuracy of miR-126. This data-driven approach may lead to an overestimation of the index test's diagnostic accuracy, especially in studies with a small sample size (27). In addition, all studies did not report whether

Table 4 Diagnostic accuracy of circulating or pleural effusion SMRP, osteopontin, fibulin-3 and mir-126 for MPM: evidence from the metaanalysis studies

\begin{tabular}{|c|c|c|c|c|c|c|}
\hline Diagnostic marker & $\mathrm{N}$ & MPM/non-MPM & Sensitivity $(95 \% \mathrm{Cl})$ & Specificity $(95 \% \mathrm{Cl})$ & AUC $(95 \% \mathrm{Cl})$ & Reference \\
\hline PE SMRP & 12 & $460 / 1,046$ & $0.79(0.75-0.83)$ & $0.85(0.83-0.87)$ & $0.89(0.85-0.93)$ & (4) \\
\hline Circulating osteopontin & 6 & $360 / 546$ & $0.65(0.60-0.70)$ & $0.81(0.78-0.85)$ & $0.83(0.79-0.87)$ & (5) \\
\hline Circulating fibulin-3 & 9 & $468 / 595$ & $0.87(0.58-0.97)$ & $0.89(0.77-0.95)$ & $0.94(0.91-0.98)$ & (7) \\
\hline Circulating miR-126 & 4 & $156 / 459$ & $0.71(0.63-0.77)$ & $0.69(0.56-0.80)$ & $0.74(0.70-0.77)$ & Present study \\
\hline
\end{tabular}

SMRP, including soluble mesothelin-related peptide; MPM, malignant pleural mesothelioma; N, number of included studies; AUC, the area under summary receiver operator characteristics (sROC) curve; $\mathrm{Cl}$, confidence interval; PE, pleural effusion. 
the control subjects received the same reference as MPM patients, indicating the possibility of partial verification bias (28).

Unlike traditional diagnostic markers such as SMRP, circulating miR-126 in MPM is lower than that of controls. The mechanisms under the decreased circulating miR126 in MPM patients remain unknown. Early studies with microRNA array indicated that miR-126 was downregulated in the tumor tissue of MPM $(20,29)$. These findings allow us to propose two possible mechanisms. The first mechanism is that circulating miR-126 is released by a special cell population. During MPM development, this cell population decreases, and thus the circulating miR126 is decreased. Another hypothesis is that during the development of MPM, the tumor promotes the absorption of circulating miR-126 by a special cell population. These two hypotheses, however, need to be validated by future studies.

Although this is the first meta-analysis investigating the diagnostic accuracy of circulating miR-126 for MPM, it has limitations. The major limitation is the small sample size, which results in wide $95 \%$ confidence intervals of sensitivity, specificity and AUC. Also, because only four studies were included, we cannot perform subgroup analysis or metaregression to explore the source of heterogeneity. Type of data collection, characteristic of control, internal control for PCR, RNA extraction methods are possible sources of heterogeneity.

Taken together, this meta-analysis suggests that the diagnostic accuracy of circulating miR-126 was low, and it is unable to replace the role of traditional markers for MPM diagnosis. Considering that some of the eligible studies have design flaws, future studies are still needed to evaluate the diagnostic accuracy of miR-126 for MPM rigorously.

\section{Acknowledgments}

Funding: This work was supported by the Natural and Science Foundation of Inner Mongolia Autonomous Region for Distinguished Young Scholars (2020JQ07).

\section{Footnote}

Reporting Checklist: The authors have completed the PRISMA-DTA reporting checklist. Available at http:// dx.doi.org/10.21037/tcr-21-104

Conflicts of Interest: All authors have completed the ICMJE uniform disclosure form (available at http://dx.doi. org/10.21037/tcr-21-104). Among all authors, only Dr. Zhi$\mathrm{De} \mathrm{Hu}$ received a funding from the Natural and Science Foundation of Inner Mongolia Autonomous Region for Distinguished Young Scholars. The other authors have no conflicts of interest to declare.

Ethical Statement: The authors are accountable for all aspects of the work in ensuring that questions related to the accuracy or integrity of any part of the work are appropriately investigated and resolved.

Open Access Statement: This is an Open Access article distributed in accordance with the Creative Commons Attribution-NonCommercial-NoDerivs 4.0 International License (CC BY-NC-ND 4.0), which permits the noncommercial replication and distribution of the article with the strict proviso that no changes or edits are made and the original work is properly cited (including links to both the formal publication through the relevant DOI and the license). See: https://creativecommons.org/licenses/by-nc-nd/4.0/.

\section{References}

1. Bibby AC, Tsim S, Kanellakis N, et al. Malignant pleural mesothelioma: an update on investigation, diagnosis and treatment. Eur Respir Rev 2016;25:472-86.

2. Gillezeau CN, van Gerwen M, Ramos J, et al. Biomarkers for malignant pleural mesothelioma: a meta-analysis. Carcinogenesis 2019;40:1320-31.

3. Niu Y, Hu ZD. Diagnostic accuracy of pleural effusion biomarkers for malignant pleural mesothelioma: a machine learning analysis. J Lab Precis Med 2021;6:4.

4. Cui A, Jin XG, Zhai K, et al. Diagnostic values of soluble mesothelin-related peptides for malignant pleural mesothelioma: updated meta-analysis. BMJ Open 2014;4:e004145.

5. Hu ZD, Liu XF, Liu XC, et al. Diagnostic accuracy of osteopontin for malignant pleural mesothelioma: a systematic review and meta-analysis. Clin Chim Acta 2014;433:44-8.

6. Otoshi T, Kataoka Y, Ikegaki S, et al. Pleural effusion biomarkers and computed tomography findings in diagnosing malignant pleural mesothelioma: A retrospective study in a single center. PLoS One 2017;12:e0185850.

7. Ren R, Yin $\mathrm{P}$, Zhang $\mathrm{Y}$, et al. Diagnostic value of fibulin-3 for malignant pleural mesothelioma: A systematic review 
and meta-analysis. Oncotarget 2016;7:84851-9.

8. Pass HI, Alimi M, Carbone M, et al. Mesothelioma Biomarkers: A Review Highlighting Contributions from the Early Detection Research Network. Cancer Epidemiol Biomarkers Prev 2020;29:2524-40.

9. Bartel DP. MicroRNAs: target recognition and regulatory functions. Cell 2009;136:215-33.

10. Bartels CL, Tsongalis GJ. MicroRNAs: novel biomarkers for human cancer. Clin Chem 2009;55:623-31.

11. Zhu HZ, Fang CJ, Guo Y, et al. Detection of miR-155-5p and imaging lung cancer for early diagnosis: in vitro and in vivo study. J Cancer Res Clin Oncol 2020;146:1941-51.

12. Bononi I, Comar M, Puozzo A, et al. Circulating microRNAs found dysregulated in ex-exposed asbestos workers and pleural mesothelioma patients as potential new biomarkers. Oncotarget 2016;7:82700-11.

13. Kirschner MB, Cheng YY, Badrian B, et al. Increased circulating miR-625-3p: a potential biomarker for patients with malignant pleural mesothelioma. J Thorac Oncol 2012;7:1184-91.

14. Han YQ, Xu SC, Zheng WQ, et al. Diagnostic value of microRNAs for malignant pleural mesothelioma: A minireview. Thorac Cancer 2021;12:8-12.

15. McInnes MDF, Moher D, Thombs BD, et al. Preferred Reporting Items for a Systematic Review and Metaanalysis of Diagnostic Test Accuracy Studies: The PRISMA-DTA Statement. JAMA 2018;319:388-96.

16. Whiting PF, Rutjes AW, Westwood ME, et al. QUADAS-2: a revised tool for the quality assessment of diagnostic accuracy studies. Ann Intern Med 2011;155:529-36.

17. Freeman SC, Kerby CR, Patel A, et al. Development of an interactive web-based tool to conduct and interrogate meta-analysis of diagnostic test accuracy studies: MetaDTA. BMC Med Res Methodol 2019;19:81.

18. Reitsma JB, Glas AS, Rutjes AW, et al. Bivariate analysis of sensitivity and specificity produces informative summary measures in diagnostic reviews. J Clin Epidemiol 2005;58:982-90.

Cite this article as: Zhang L, Zhou Q, Han YQ, Li P, Ouyang PH, Wang MY, Hu ZD. Diagnostic accuracy of circulating miR-126 for malignant pleural mesothelioma: a systematic review and meta-analysis. Transl Cancer Res 2021;10(4):1856-1862. doi: $10.21037 /$ tcr-21-104
19. Walter SD. Properties of the summary receiver operating characteristic (SROC) curve for diagnostic test data. Stat Med 2002;21:1237-56.

20. Santarelli L, Strafella E, Staffolani S, et al. Association of MiR-126 with soluble mesothelin-related peptides, a marker for malignant mesothelioma. PLoS One 2011;6:e18232.

21. Tomasetti M, Staffolani S, Nocchi L, et al. Clinical significance of circulating miR-126 quantification in malignant mesothelioma patients. Clin Biochem 2012;45:575-81.

22. Santarelli L, Staffolani S, Strafella E, et al. Combined circulating epigenetic markers to improve mesothelin performance in the diagnosis of malignant mesothelioma. Lung Cancer 2015;90:457-64.

23. Weber DG, Gawrych K, Casjens S, et al. Circulating miR132-3p as a Candidate Diagnostic Biomarker for Malignant Mesothelioma. Dis Markers 2017;2017:9280170.

24. Zhang M, Hu ZD. Suggestions for designing studies investigating diagnostic accuracy of biomarkers. Ann Transl Med 2019;7:788.

25. Bossuyt PM, Reitsma JB, Bruns DE, et al. Towards complete and accurate reporting of studies of diagnostic accuracy: the STARD initiative. Standards for Reporting of Diagnostic Accuracy. Clin Chem 2003;49:1-6.

26. Rutjes AW, Reitsma JB, Vandenbroucke JP, et al. Casecontrol and two-gate designs in diagnostic accuracy studies. Clin Chem 2005;51:1335-41.

27. Leeflang MM, Moons KG, Reitsma JB, et al. Bias in sensitivity and specificity caused by data-driven selection of optimal cutoff values: mechanisms, magnitude, and solutions. Clin Chem 2008;54:729-37.

28. de Groot JA, Bossuyt PM, Reitsma JB, et al. Verification problems in diagnostic accuracy studies: consequences and solutions. BMJ 2011;343:d4770.

29. Andersen M, Grauslund M, Ravn J, et al. Diagnostic potential of miR-126, miR-143, miR-145, and miR652 in malignant pleural mesothelioma. J Mol Diagn 2014;16:418-30. 